\title{
Palpation thyroiditis following subtotal parathyroidectomy for hyperparathyroidism
}

\author{
Elizabeth M Madill1, Shamil D Cooray¹ and Leon A Bach1,2 \\ 'Department of Endocrinology and Diabetes, The Alfred Hospital, Melbourne, Victoria, Australia and \\ 2Department of Medicine (Alfred), Monash University, Melbourne, Victoria, Australia
}

Correspondence should be addressed to L A Bach

Email

leon.bach@monash.edu

\section{Summary}

Thyrotoxicosis is an under-recognised but clinically important complication of parathyroidectomy. We report a case of a 37-year-old man with tertiary hyperparathyroidism who initially developed unexplained anxiety, diaphoresis, tachycardia, tremor and hyperreflexia one day after subtotal parathyroidectomy. Thyroid biochemistry revealed suppressed thyroid stimulating hormone and elevated serum free $T_{4}$ and free $T_{3}$ levels. Technetium- $99 \mathrm{~m}$ scintigraphy scan confirmed diffusely decreased radiotracer uptake consistent with thyroiditis. The patient was diagnosed with thyrotoxicosis resulting from palpation thyroiditis. Administration of oral beta-adrenergic antagonists alleviated his symptoms and there was biochemical evidence of resolution fourteen days later. This case illustrates the need to counsel patients about thyroiditis as one of the potential risks of parathyroid surgery. It also emphasises the need for biochemical surveillance in patients with unexplained symptoms in the post-operative period and may help to minimise further invasive investigations for diagnostic clarification.

\section{Learning points:}

- Thyroiditis as a complication of parathyroidectomy surgery is uncommon but represents an under-recognised phenomenon.

- It is thought to occur due to mechanical damage of thyroid follicles by vigorous palpation.

- Palpation of the thyroid gland may impair the physical integrity of the follicular basement membrane, with consequent development of an inflammatory response.

- The majority of patients are asymptomatic, however clinically significant thyrotoxicosis occurs in a minority.

- Patients should be advised of thyroiditis/thyrotoxicosis as a potential complication of the procedure.

- Testing of thyroid function should be performed if clinically indicated, particularly if adrenergic symptoms occur post-operatively with no other cause identified.

\section{Background}

Although thyroiditis can occur following parathyroidectomy surgery, it is not a commonly recognised clinical complication. This phenomenon has been described in the literature as 'palpation thyroiditis' (1). The mechanism has not been fully elucidated but several theories have been proposed. Intraoperative trauma by vigorous palpation of the gland causing rupture of isolated thyroid follicles is the most commonly postulated mechanism. The risk appears to increase with the extent of neck exploration surgery $(2,3)$, but palpation thyroiditis has also been described in patients undergoing surgery for primary hyperparathyroidism $(4,5)$. Palpation of the thyroid gland causing impairment of the physical integrity of the follicular epithelium and a subsequent 
inflammatory process otherwise termed 'multifocal granulomatous folliculitis' has also been hypothesised as a causal mechanism (4). Histologically this is characterised by an infiltrate with histiocytes, plasma cells, lymphocytes and haemosiderin-laden macrophages (6).

The majority of patients with palpation thyroiditis are clinically asymptomatic and only a minority develop symptoms of overt thyrotoxicosis. The incidence of TSH suppression in clinically asymptomatic patients is much higher. Studies have reported biochemical hyperthyroidism, defined as full TSH suppression, occurring after parathyroidectomy in as many as $31-42 \%$ of patients, whereas only $\sim 15 \%$ are symptomatic (5). The variability in incidence rates may be accounted for by (i) the primary indication for surgery; (ii) varying operative techniques used for primary, secondary and tertiary hyperparathyroidism; (iii) the extent of intraoperative gland manipulation; and (iv) variations in thyroid shape, size and pre-operative function (5).

Abnormal values of TSH, free $\mathrm{T}_{4}\left(\mathrm{fT}_{4}\right)$ and free $\mathrm{T}_{3}\left(\mathrm{fT}_{3}\right)$ indicating post-operative thyrotoxicosis are observed in patients with palpation thyroiditis. The levels of $\mathrm{fT}_{4}$ and $\mathrm{fT}_{3}$ usually reach their maximum on post-operative day $1-3$, whereas the TSH nadir occurs around post-operative day 5 (5). Thyroglobulin released from the injured thyroid follicles also increases, and has been suggested as a suitable marker supporting the diagnosis. Thyroglobulin levels greater than $90 \mathrm{ng} / \mathrm{mL}$ are suggestive of post-operative palpation thyroiditis (5). Spontaneous resolution can be expected by 6 weeks in the vast majority of patients given that the half-life of $\mathrm{fT}_{4}$ is one week (i.e. 5-6 halflives until resolution) (3). Persistence thereafter should prompt the clinician to search for an alternative cause of hyperthyroidism.

We report a case of a patient who underwent subtotal parathyroidectomy for tertiary hyperparathyroidism, which was complicated by thyroiditis causing symptomatic thyrotoxicosis. This is the first published case report of such early thyrotoxicosis as supported by a TSH nadir by day 5 post-operatively and absence of tracer uptake on a Tc-99m pertechnetate thyroid scan.

\section{Case presentation}

A 37-year-old man with tertiary hyperparathyroidism in the setting of end-stage renal failure secondary to hypertension and a unilateral kidney was electively admitted for a subtotal parathyroidectomy. He had no history of thyroid disease and had not received any iodinated contrast agents or iodine-containing medications before the operation. He gave no history of neck pain, recent fever or neck irradiation. There was no family history of thyroid disease.

Pre-operatively he was biochemically euthyroid with serum TSH $1.38 \mathrm{mU} / \mathrm{L}$ (reference range: 0.30-5.00), $\mathrm{fT}_{4} 12.0 \mathrm{pmol} / \mathrm{L}$ (reference range, 9.1-19.6) and $\mathrm{fT}_{3}$ $1.8 \mathrm{pmol} / \mathrm{L}$ (reference range: 2.4-5.9). As part of the preoperative workup the patient also underwent a thyroid ultrasound demonstrating a mildly heterogeneous nodular appearance of both lobes. The right lobe measured $16 \times 16 \times 51 \mathrm{~mm}$ and the left lobe measured $17 \times 10 \times 44 \mathrm{~mm}$. The largest nodule in the left thyroid lobe was a well-circumscribed $19 \times 12 \times 12 \mathrm{~mm}$ lesion at the junction with the isthmus. This was biopsied and the histopathology was consistent with a benign colloid cyst. Within the right thyroid lobe, there were two nodules, one measuring $5 \times 5 \times 9 \mathrm{~mm}$ and the other measuring $7 \times 6 \times 21 \mathrm{~mm}$. Both had similar appearances and were suggestive of parathyroid adenomas. The patient went on to have a sestamibi scan, which suggested multinodular changes but no scintigraphic evidence of a parathyroid adenoma.

He underwent subtotal parathyroidectomy. There was difficulty with dissection and mobilisation of the left inferior parathyroid gland, which was densely adhering to the surrounding tissues. In total, three and a half parathyroid glands were excised and the total operative time was approximately one and a half hours. Histopathology confirmed the presence of parathyroid tissue with uniformly round typical nuclei and no areas of necrosis or fibrous septae.

On post-operative day 1 , the patient developed irritability, palpitations, insomnia and vivid dreams. Physical examination revealed signs of thyrotoxicosis including diaphoresis, a resting sinus tachycardia with a heart rate of $130-150$ beats per minute, bilateral hand tremor and globally brisk reflexes. There was no goitre, neck tenderness, thyroid bruit or signs of thyroid ophthalmopathy.

Laboratory investigations were consistent with thyrotoxicosis with a partially suppressed serum TSH of $0.16 \mathrm{mU} / \mathrm{L}$ (reference range: 0.30-5.00), elevated $\mathrm{fT}_{4}$ of $37.8 \mathrm{pmol} / \mathrm{L}$ (reference range: 9.1-19.6) and $\mathrm{fT}_{3}$ of $5.5 \mathrm{pmol} / \mathrm{L}$ (reference range: 2.4-5.9), and elevated thyroglobulin of $255.4 \mu \mathrm{g} / \mathrm{L}$ (reference range: 3.5-77). Antithyroid peroxidase antibody testing was negative. The TSH became completely suppressed by the post-operative 
day 5 with a persistently elevated $\mathrm{fT}_{4}$ of $22.6 \mathrm{pmol} / \mathrm{L}$ and normal $\mathrm{fT}_{3}$ of $3.3 \mathrm{pmol} / \mathrm{L}$.

A Tc-99m pertechnetate thyroid scan showed diffusely decreased radiotracer uptake throughout both lobes of the thyroid gland with overall thyroid gland uptake significantly lower than background salivary gland uptake. The features overall were in keeping with thyroiditis (Fig. 1).

\section{Investigations}

Refer to the preceding case presentation for biochemical results.

\section{Treatment}

The patient was treated symptomatically with up-titration of his regular dose of metoprolol from $50 \mathrm{mg}$ BD to $150 \mathrm{mg}$ BD with rapid and good effect.

\section{Outcome and follow-up}

Fourteen days after the operation, the patient was reviewed in the outpatient clinic with repeat laboratory investigations. These demonstrated complete resolution with TSH $2.11 \mathrm{mU} / \mathrm{L}$ (reference range: 0.30-5.00), $\mathrm{fT}_{4}$ $16.6 \mathrm{pmol} / \mathrm{L}$ (reference range: 9.1-19.6), $\mathrm{fT}_{3} 4.7 \mathrm{pmol} / \mathrm{L}$ (reference range: $2.4-5.9$ ) and thyroglobulin $21.2 \mu \mathrm{g} / \mathrm{L}$
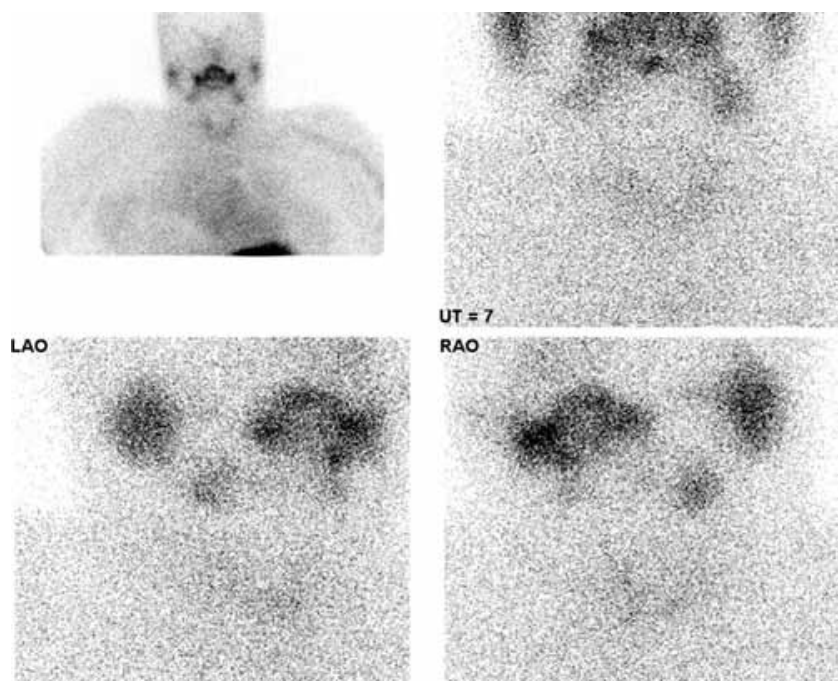

\section{Figure 1}

Technetium-99m scintigraphy scan of the thyroid gland. The radiotracer uptake is diffusely decreased throughout both lobes of the thyroid gland with overall thyroid uptake significantly less than background salivary gland uptake. Features are in keeping with thyroiditis. (reference range, 3.5-77). His metoprolol dose was reduced to baseline $50 \mathrm{mg}$ BD. Subsequent thyroid function tests 4 weeks later showed he remained euthyroid, and there was no documented hypothyroid phase of his illness.

\section{Discussion}

Palpation thyroiditis causes transient hyperthyroidism and can occur as a complication of surgery for hyperparathyroidism. It reflects release of preformed hormone from damaged follicular cells, but may also occur because of alteration in the integrity of the follicular basement membrane. Biochemically, a large proportion of patients are hyperthyroid following surgery; however, a much smaller proportion of patients are symptomatic. This may suggest the possibility of under-diagnosis due to the high prevalence of asymptomatic disease. This may have clinical implications such as exacerbation of pre-existing coronary heart disease or precipitation of atrial arrhythmias (7). In a prospective study by Stang et al., the majority of 125 patients had biochemical evidence of hyperthyroidism after parathyroidectomy, and the majority of them were asymptomatic or mildly symptomatic (3).

Rudofsky et al. (7) evaluated 39 patients undergoing surgery for secondary hyperparathyroidism in the context of end-stage renal failure with pre- and post-operative thyroid function tests. Thirty of the 39 patients with normal preoperative serum TSH, $\mathrm{fT}_{4}$ and $\mathrm{fT}_{3}$ displayed post-operative biochemical evidence of hyperthyroidism, and $60 \%$ of these patients were symptomatic. Maximum levels of $\mathrm{fT}_{4}$ and $\mathrm{fT}_{3}$ were reached on post-operative days $1-3$, and the TSH nadir was reached by day 5 . By day 40 , there was spontaneous resolution of clinical symptoms and biochemical normalisation in all cases with no significant long-term sequelae reported.

We report a case of a man with significant symptoms consistent with overt thyrotoxicosis in the immediate days after successful and otherwise uncomplicated parathyroid surgery. Suppressed TSH, elevated $\mathrm{fT}_{4}, \mathrm{fT}_{3}$ and thyroglobulin levels and reduced scan uptake aided in diagnostic clarification. Thyrotoxicosis in our patient is suspected to have resulted from intraoperative palpation of the gland resulting in palpation thyroiditis.

As demonstrated, there is potential for harm, particularly in the early period, in patients who develop overt thyrotoxicosis following parathyroid surgery who are undiagnosed and left untreated. When necessary, beta-adrenergic antagonists may be required to control symptoms of overt thyrotoxicosis and prevent further 
deterioration. Awareness of this complication of surgery is also important to avoid unnecessary and potentially harmful investigations and treatments to patients with otherwise unexplained adrenergic symptoms in the post-operative period. Clinicians should be encouraged to counsel patients about thyroiditis and thyrotoxicosis as potential risks following parathyroid surgery and to perform routine biochemical surveillance if clinically indicated.

\section{Declaration of interest}

There is no conflict of interest that could be perceived as prejudicing the impartiality of the research reported.

\section{Funding}

This research did not receive any specific grant from any funding agency in the public, commercial or not-for-profit sector.

\section{Patient consent}

Written informed consent was obtained from the patient for publication of this case report.

Author contributions and acknowledgements

Dr Elizabeth Madill - Basic Physician Trainee, worked with the Department of Renal Medicine at Alfred Health and looked after the patient while he was an inpatient and was the principal author of this manuscript. Dr Shamil Cooray - Advanced Trainee in Endocrinology, worked with the Department of Endocrinology and Diabetes at Alfred Health, reviewed the patient in outpatient clinic, and assisted $\mathrm{Dr}$ Madill with the literature review and in editing of the case report and discussion. Prof Leon Bach - Consultant Endocrinologist and Deputy Director of the Department of Endocrinology and Diabetes was the treating endocrinologist during his admission and supervised Dr Madill in editing of the case report and discussion.

\section{References}

1 Hwang TS \& Park SH 1988 Histopathological study of the so called 'palpation thyroditis'. Journal of Korean Medical Science 3 27-29. (doi:10.3346/jkms.1988.3.1.27)

2 Lederer SR \& Schiffl H 2008 Transient hyperthyroidism after total parathyroidectomy for hyperparathyroidism: a report of two cases. Wien Klin Wochenschr 14 432-434. (doi:10.1007/s00508-008-0996-3)

3 Stang MT, Yim JH, Challinor SM, Bahl S \& Carty SE 2005 Hyperparathyroidism after parathyroid exploration. Surgery 138 1058-1065. (doi:10.1016/j.surg.2005.09.011)

4 Espiritu R \& Dean D 2010 Parathyroidectomy induced thyroiditis. Endocrine Practice 16 656-659 (doi:10.4158/EP09367.CR)

5 Walfish PG, Caplan D \& Rosen IB 1992 Post-parathyroidectomy transient thyrotoxicosis. Journal of Clinical Endocrinology and Metabolism 75 224-227. (doi:10.1210/jcem.75.1.1619014)

6 Carney JA, Moore SB \& Northcutt RC 1975 Palpation thyroiditis (multifocal granulomatous folliculitis). American Journal of Clinical Pathology 64 639-647. (doi:10.1093/ajcp/64.5.639)

7 Rudofsky G, Tsioga M, Leowardi C, Kopf S, Grafe A, Mawroth PP \& Isermann B Transient hyperthyroidism after surgery for secondary hyperparathyroidism: a common problem. European Journal of Medical Research 201116 375-380. (doi:10.1186/2047783X-16-8-375)

Received in final form 16 June 2016

Accepted 1 July 2016 